\title{
AIP
}

\section{Thermoelectric properties of DC-sputtered filled skutterudite thin film}

Gaosheng Fu, Lei Zuo, Jie Chen, Ming Lu, and Liangyao Yu

Citation: Journal of Applied Physics 117, 125304 (2015); doi: 10.1063/1.4916238

View online: http://dx.doi.org/10.1063/1.4916238

View Table of Contents: http://scitation.aip.org/content/aip/journal/jap/117/12?ver=pdfcov

Published by the AIP Publishing

\section{Articles you may be interested in}

Preparation and thermoelectric properties of B4C-Si-B composites

AIP Conf. Proc. 1449, 315 (2012); 10.1063/1.4731560

Thermoelectric properties of indium filled and germanium doped Co4Sb12 skutterudites

J. Appl. Phys. 111, 023708 (2012); 10.1063/1.3677982

Dynamic in situ observations of electrical and structural changes in thin thermoelectric ( $\mathrm{Bi} 0.15 \mathrm{Sb} 0.85$ ) 2 Te 3 films

J. Appl. Phys. 106, 063711 (2009); 10.1063/1.3225610

Thermoelectric properties of $\mathrm{n}$-type nanocrystalline bismuth-telluride-based thin films deposited by flash evaporation

J. Appl. Phys. 101, 074301 (2007); 10.1063/1.2717867

Transport and structural properties of binary skutterudite CoSb 3 thin films grown by dc magnetron sputtering technique

J. Appl. Phys. 92, 5319 (2002); 10.1063/1.1513188

MIT LINCOLN

LABORATORY CAREERS

Discover the satisfaction of innovation and service

to the nation
- Space Control

- Air \& Missile Defense

- Communications Systems \& Cyber Security

- Intelligence, Surveillance and

Reconnaissance Systems

- Advanced
Electronics
- Tactical Systems
- Homeland
Protection
- Air Traffic Control

LINCOLN LABORATORY

MassachusetTs Institute of TeChNOLOgY

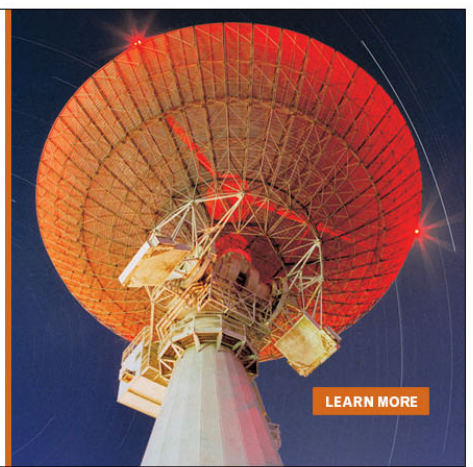




\title{
Thermoelectric properties of DC-sputtered filled skutterudite thin film
}

\author{
Gaosheng Fu, ${ }^{1}$ Lei Zuo, ${ }^{1,2, a)}$ Jie Chen, ${ }^{2}$ Ming Lu, ${ }^{3}$ and Liangyao $\mathrm{Yu}^{4}$ \\ ${ }^{1}$ Department of Mechanical Engineering, Stony Brook University, Stony Brook, New York 11794, USA \\ ${ }^{2}$ Department of Mechanical Engineering, Virginia Tech, Blacksburg, Virginia 24061, USA \\ ${ }^{3}$ Center for Functional Nanomaterials, Brookhaven National Laboratory, Upton, New York 11973, USA \\ ${ }^{4}$ State Key Laboratory of Automotive Safety and Energy, Tsinghua University, Beijing 10084, China
}

(Received 25 November 2014; accepted 14 March 2015; published online 25 March 2015)

\begin{abstract}
The $\mathrm{Yb}$ filled $\mathrm{CoSb}_{3}$ skutterudite thermoelectric thin films were prepared by DC magnetron sputtering. The electrical conductivity, Seebeck coefficient, thermal conductivity, and figure of merit $Z T$ of the samples are characterized in a temperature range of $300 \mathrm{~K}$ to $700 \mathrm{~K}$. X-ray diffraction, scanning electron microscopy, and energy-dispersive X-ray spectroscopy are obtained to assess the phase composition and crystallinity of thin film samples at different heat treatment temperatures. Carrier concentrations and Hall mobilities are obtained from Hall Effect measurements, which provide further insight into the electrical conductivity and Seebeck coefficient mechanisms. The thermal conductivity of thin film filled skutterudite was found to be much less compared with bulk Yb filled $\mathrm{CoSb}_{3}$ skutterudite. In this work, the $1020 \mathrm{~K}$ heat treatment was adopted for thin film post process due to the high degree of crystallinity as well as avoiding reverse heating effect. Thin film samples of different thicknesses were prepared with the same sputtering deposition rate and maximum $Z T$ of 0.48 was achieved at $700 \mathrm{~K}$ for the $130 \mathrm{~nm}$ thick sample. This value was between half and one third of the bulk figure of merit which was due to the lower Hall mobility. (C) 2015 AIP Publishing LLC. [http://dx.doi.org/10.1063/1.4916238]
\end{abstract}

\section{INTRODUCTION}

Thermoelectric generators allow the direct solid-state conversion between thermal and electrical energy, ${ }^{1}$ which are of particular interest for waste heat energy harvesting, ${ }^{2}$ energy efficiency improvement, ${ }^{3}$ and powering of remote sensors and electronic applications. ${ }^{4}$ Among the many potential applications, thermoelectrics for power generation or temperature control in micro-electronic device ${ }^{5}$ are especially attractive since heat plays a key role to degrade the device performance. ${ }^{6}$

Conventional thermoelectric modules, sometimes referred to as "bulk" modules, have been used for decades in commercial power generation (ex, Hi-z) $)^{7}$ and temperature control of electronics. ${ }^{8}$ However, as the size and power density requirements are dramatically changing for electronics, conventional bulk thermoelectric technology will lose its advantages for the microelectronic applications. Conversely, thin film thermoelectric modules focus on localized temperature control as well as self-sustained power generation. ${ }^{9,10}$ These thin film thermoelectric modules take the advantages of semiconductor processing techniques to create a nanostructured thermoelectric thin film. ${ }^{11}$ As the Internet of Things (IoT) develops, more embedded thermoelectric energy harvesting modules are needed for a variety of applications due to its advantage of compactness and free of maintenance compared with using regular battery. ${ }^{12}$ The current type of the thin film thermoelectric module that commercially available is based on bismuth telluride alloy which has good performance at room temperature. ${ }^{13}$ For energy

\footnotetext{
a) Author to whom correspondence should be addressed. Electronic mail: leizuo@vt.edu
}

harvesting applications in nuclear or other harsh environments, electronic systems must survive high temperatures and thermoelectric devices based on high temperature materials other than bismuth telluride are desirable.

Filled skutterudite is a state of the art high temperature thermoelectric material which is known as a phonon glass electron crystal-PGEC system. ${ }^{14}$ Nolas et al. ${ }^{15}$ reported on bulk $\mathrm{Yb}$ filled skutterudite and found $Z T$ close to 1 at $600 \mathrm{~K}$ for $\mathrm{Yb}_{0.19} \mathrm{Co}_{4} \mathrm{Sb}_{12}$. Shi et al. ${ }^{16}$ investigated multi-filled skutterudite bulk material and got maximum ZT of 1.7 at $850 \mathrm{~K}$. For microdevice applications, ${ }^{17}$ it is essential to find these promising "bulk" properties also in skutterudite thin films. Thermoelectric modules based on thin film skutterudite material are still under laboratory research level. ${ }^{18,19}$ Anno et al. ${ }^{20,21}$ reported the grown of $\mathrm{CoSb}_{3}$ thin film on $\mathrm{Si}(100)$ and GaAs (100) substrate using rf-sputtering. In another study, Savchuk et al. ${ }^{22}$ studied the structural, electrical, and thermal properties of binary skutterudite $\mathrm{CoSb}_{3}$ thin films deposited using sputtering. The temperature of the transition from the amorphous to crystalline state was found to be around $428 \mathrm{~K}$. On the other hand, Caylor et al. ${ }^{23}$ presented skutterudite $\left(\mathrm{CoSb}_{3}\right.$ and $\left.\mathrm{IrSb}_{3}\right)$ thin films of high phase purity and crystallinity. Zeipl et al. ${ }^{24,25}$ studied thin layers of $\mathrm{Yb}$ filled $\mathrm{CoSb}_{3}$ prepared by pulsed laser deposition (PLD) with the best results obtained on quartz substrate at $470 \mathrm{~K}$. Kumar et al. ${ }^{26}$ reported In and Yd double filled $\mathrm{CoSb}_{3}$ thin film using PLD and obtained maximum power factor of $0.68 \mathrm{~W} / \mathrm{m} \mathrm{K}^{2}$ at $700 \mathrm{~K}$. However, all the studies reported on thin film skutterudite did not provide the figure of merit at high temperatures which impeded the development for high temperature applications.

In this work, the preparation of $\mathrm{Yb}$ filled skutterudite thin film growth using DC magnetron sputtering is summarized 
and thermoelectric properties including thermal conductivity, electrical conductivity, Seebeck coefficient, and figure of merit are reported from room temperature to $700 \mathrm{~K}$. The selection of using DC magnetron sputtering lies in a high deposition rate and it also maintains the target material composition. ${ }^{27}$ More importantly, magnetron sputtering produces a dense microstructure which surpasses most of other thin film deposition techniques. ${ }^{28}$ The parameters of DC magnetron sputtering and post heat treatment on sputtered sample have been optimized experimentally. $\mathrm{Yb}$ filled $\mathrm{CoSb}_{3}$ has for the first time been successfully deposited using sputtering and characterized comprehensively. This result is reported in the following manner: Section II introduces the experiment method used in this work; Sec. III discusses in detail about the phase, microstructure, and thermoelectric properties; Sec. IV concludes the work of $\mathrm{Yb}$ filled skutterudite prepared with of DC magnetron sputtering.

\section{METHODS}

Thermoelectric performance is characterized by the dimensionless figure of merit $Z T=\sigma S^{2} T / \kappa$ and power factor $P F=\sigma S^{2},{ }^{29}$ where $S$ is the Seebeck coefficient $(\mathrm{V} / \mathrm{K}), \sigma$ is the electrical conductivity $(\mathrm{S} / \mathrm{m}), \kappa$ is the thermal conductivity $(\mathrm{W} / \mathrm{m} \mathrm{K})$, and $\mathrm{T}$ is the absolute temperature in Kevin (K). The electrical conductivity $\sigma=n e \mu$ is given by carrier concentration $n$, carrier mobility $\mu$, and electron charge $e$. Carrier concentration and mobility can be determined by measuring the Hall coefficient $R_{H}$. For materials in which electrons are the primary carriers, $R_{H}=-1 / n e$. Combining the Hall Effect coefficient and electrical conductivity, the mobility of such materials can be expressed as $\mu=-\sigma R_{H}$. An expression for the Seebeck coefficient for $n$-type thermoelectric material is obtained by Busch and Winkler ${ }^{30}$ as

$$
S=-\frac{k}{e}\left(\frac{5}{2}+r-\ln \frac{n}{N_{c}}\right) .
$$

Here, $k$ is Boltzmann's constant, $r$ is the exponent of the power function in the energy-dependent relaxation time expression, ${ }^{31}$ and $N_{c}$ is the effective density of states in the valence band. As can be seen, the Seebeck coefficient is affected by carrier concentration, $n$.

Used in this study are four-inch-diameter undoped $\langle 100\rangle$ silicon wafers (University Wafer, Inc.). The 350- to $400-\mu$ m-thick silicon wafers with a resistivity of more than $1 \mathrm{M} \Omega \mathrm{cm}$ were initially oxidized at $670 \mathrm{~K}$ for 2 minutes to get a 240-nm-thick silicon dioxide layer using pressure enhanced chemical vapor deposition PECVD (Trion Technology, Inc.). The target material is purchased from LTS Research Laboratories, Inc. The films were deposited at room temperature without substrate heating.

After pumping overnight, the process chamber had a base pressure of $5 \times 10^{-6}$ Torr. The thickness of thin films was controlled by a film thickness monitor/controller during the deposition. Table I summaries some of the parameters used in the sputtering process.

Table II summarizes all of the samples in this work including target and sputtered samples as well as their heat
TABLE I. DC magnetron sputtering parameters.

\begin{tabular}{lc}
\hline \hline Sputtering gas & $\mathrm{Ar}$ \\
Sputtering power (W) & 100 \\
Base pressure (Torr) & $5 \times 10^{-6}$ \\
Total gas pressure (Torr) & $5 \times 10^{-3}$ \\
Substrate temperature (Ts) & Room temperature \\
Substrate to target distance (cm) & 40 \\
Substrate material & Oxidized silicon \\
Deposition rate (nm/s) & 0.2 \\
\hline
\end{tabular}

treated counterparts. The thickness of the film is measured by Stylus Profilometry (Bruker, IL, USA). Target material T1 is as-quenched filled skutterudite and T2 is a sample heat treated at $1020 \mathrm{~K}$ for $120 \mathrm{~h} \mathrm{Y1}$ is as sputtered filled skutterudite thin film and Y2 through Y6 are the several thicknesses thin film samples with various heat treatments.

The sample phase was analyzed by X-ray diffraction (XRD) (Ultimate III, Rigaku, TX, USA) utilizing $\mathrm{Cu} \mathrm{K} \alpha$ radiation and energy-dispersive X-ray spectroscopy (EDS) (JEOL $7600 \mathrm{~F}$, Tokyo, JP). The surface morphologies of the sample were observed using a scanning electric microscope (SEM) (JEOL 7600F, Tokyo, JP). The electrical conductivity was measured using a Keithley digital multimeter (Model 2700, Keithley Instruments, Inc., OH, USA) and the sample was heated from 300 to $400 \mathrm{~K}$ using a High Temperature Probe Station (MBE-Komponenten AO500, Germany). To investigate their contribution to electrical conductivity, the carrier concentration and Hall mobility were measured with a Hall Measurement System (8404, Lake Shore Cryotronics, Inc., $\mathrm{OH}, \mathrm{USA}$ ) using the Van der Pauw method. Seebeck coefficients were measured using a differential method in vacuum. The thermal conductivity was measured using $3 \omega$ method by a lock-in amplifier together with temperature coefficient of resistance (TCR) in a high temperature probe station.

\section{RESULTS AND DISCUSSIONS}

\section{A. Phase composition of filled skutterudite thin film before and after heat treatment}

Fig. 1 shows the XRD of the DC magnetron sputtered thin film as well as targets before and after heat treatment. As can been seen from the figure, the as quenched target (T1) basically contains $\mathrm{Sb}$ and $\mathrm{CoSb}_{2}$ phases, instead of a $\mathrm{CoSb}_{3}$ phase. The reason to choose "as quenched" target is a

TABLE II. Sample synthesis method and post process.

\begin{tabular}{lcccc}
\hline \hline Sample & Material & Condition & Heat treatment & Thickness (nm) \\
\hline $\mathrm{T} 1$ & $\mathrm{Yb}_{\mathrm{x}} \mathrm{CoSb}_{3}$ & Hot press & N/A & Target \\
$\mathrm{T} 2$ & $\mathrm{Yb}_{\mathrm{x}} \mathrm{CoSb}_{3}$ & Hot press & $1020 \mathrm{~K}, 120 \mathrm{~h}$ & Target \\
$\mathrm{Y} 1$ & $\mathrm{Yb}_{\mathrm{x}} \mathrm{CoSb}_{3}$ & Sputtering & N/A, as deposited & 860 \\
$\mathrm{Y} 2$ & $\mathrm{Yb}_{\mathrm{x}} \mathrm{CoSb}_{3}$ & Sputtering & $620 \mathrm{~K}, 2 \mathrm{~h}$ & 860 \\
$\mathrm{Y} 3$ & $\mathrm{Yb}_{\mathrm{x}} \mathrm{CoSb}_{3}$ & Sputtering & $870 \mathrm{~K}, 4 \mathrm{~h}$ & 860 \\
$\mathrm{Y} 4$ & $\mathrm{Yb}_{\mathrm{x}} \mathrm{CoSb}_{3}$ & Sputtering & $1020 \mathrm{~K}, 24 \mathrm{~h}$ & 860 \\
$\mathrm{Y} 5$ & $\mathrm{Yb}_{\mathrm{x}} \mathrm{CoSb}_{3}$ & Sputtering & $1020 \mathrm{~K}, 24 \mathrm{~h}$ & 300 \\
$\mathrm{Y} 6$ & $\mathrm{Yb}_{\mathrm{x}} \mathrm{CoSb}_{3}$ & Sputtering & $1020 \mathrm{~K}, 24 \mathrm{~h}$ & 130 \\
\hline \hline
\end{tabular}



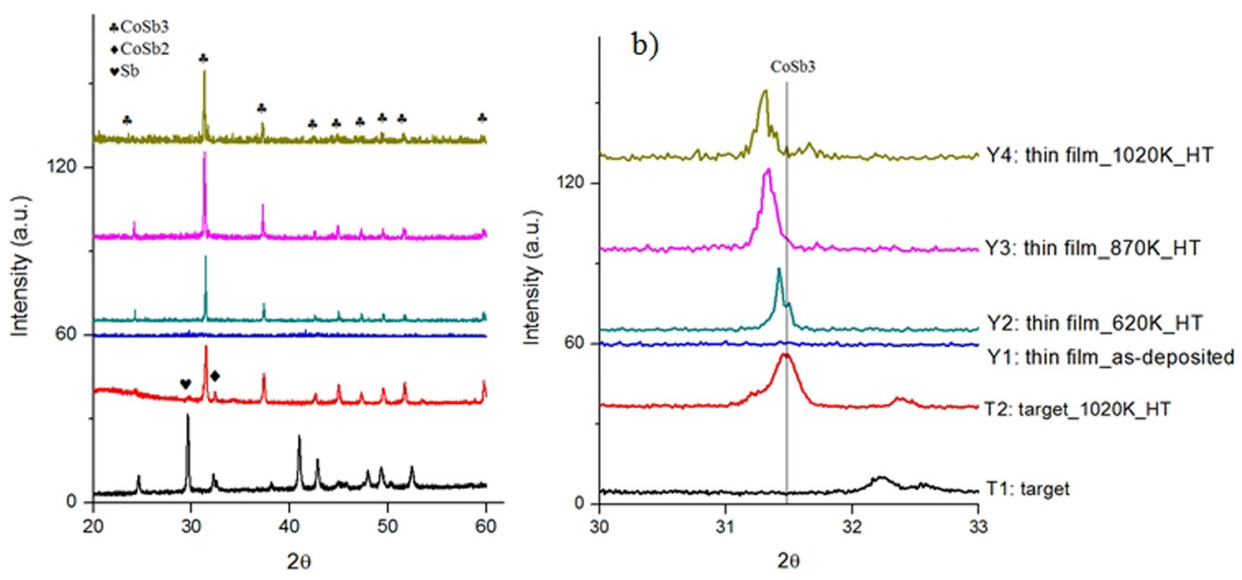

FIG. 1. XRD of DC magnetron sputtered filled skutterudite thin film and target material with different heat treatment with $2 \theta$ of (a) 20 to 80 degree and (b) 30 to 33 degree.

consideration of production availability and price. Heat treatment on the "as quenched" target material at $1020 \mathrm{~K}$ (T2) gives mainly $\mathrm{CoSb}_{3}$ phase with a little residual $\mathrm{CoSb}_{2}$ phase. The as-sputtered thin film (Y1) is measured as amorphous due to the fact that the substrate temperature during sputtering is kept at room temperature. When heat treated at $620 \mathrm{~K}$ (Y2), the thin film turns from amorphous into crystalline. The crystallized thin film shows a main peak of $\mathrm{CoSb}_{3}$ which is quite different from the bulk material since bulk filled skutterudite needs higher and longer temperatures to be fully turned into $\mathrm{CoSb}_{3}$ phase. The reason for this is that the thin film after the sputtering process is more uniform and the diffusion occurs more readily due to the lower dimension in thin film sample. Higher temperature heat treatment on $870 \mathrm{~K}$ (Y3) and $1020 \mathrm{~K}$ (Y4) can make the film fully crystalline and possibly facilitate the $\mathrm{Yb}$ diffusion into the $\mathrm{CoSb}_{3}$ cages which is shown in Fig. 1(b). Filled skutterudite and skutterudite $\left(\mathrm{CoSb}_{3}\right)$ are the similar phases and the only difference is the left shift of the peaks in filled skutterudites. As shown in Fig. 1(b), thin film skutterudite clearly shows the shift of peaks which is originated from the diffusion of $\mathrm{Yb}$ element into $\mathrm{CoSb}_{3}$ cages. After the diffusion of $\mathrm{Yb}$ element into $\mathrm{CoSb}_{3}$ cage, the lattice constant of the sample will increase and the peak shown on XRD graph will shift towards left (smaller $\theta$ ). As temperature increases in thin film sample heat treatment, more shift of XRD peak is found which is due to the enhanced diffusion of $\mathrm{Yb}$ element into $\mathrm{CoSb}_{3}$ cage at higher temperature. The confirmation of $\mathrm{Yb}$ element from EDS (Fig. 2) together with the lattice structure changed (Fig. 1(b)) suggested the Yb filled skutterudite phase is formed in thin film sample after proper heat treatment. Overall, after $620 \mathrm{~K}$ heat treatment, the thin film turns from amorphous into crystalline and $1020 \mathrm{~K}$ with $24 \mathrm{~h}$ heat treatment gives best electrical properties as shown in Table III.

In Fig. 1(b), the change of lattice structure has been shown due to the shift of XRD peak. But the trace Yb element is not found in the XRD result due to its low concentration. The EDS is used to check the presence of Yb element as well as the relative percentage through the semiquantitative analysis. The EDS analysis (Fig. 2) shows the similar $\mathrm{Yb}$ content in the thin film Y1 and $\mathrm{Y} 4$ which is about 1 at. \% corresponding to $\mathrm{Yb}_{0.15} \mathrm{Co}_{4} \mathrm{Sb}_{12}$ giving $\mathrm{N}$ type electrical property.

\section{B. SEM results of dc-sputtered filled skutterudite thin film samples}

Heat treatment is necessary for filled skutterudite samples in order to get the correct phase and better thermoelectric properties. As shown in Fig. 3(a), the as-deposited material is amorphous because during sputtering the substrate is at room temperature. This is typically true for thin film grown through sputtering. A $620 \mathrm{~K}$ heat treatment for 2 $\mathrm{h}$ (Y2) turns the thin film into crystal as one can see from above XRD (Fig. 1). Figs. 3(c) and 3(d) show that higher temperature heat treatment gives larger grain size which will enhance Hall mobility as shown in Table III.

\section{Thermoelectric properties of dc-sputtered filled skutterudite thin film samples}

\section{Electrical conductivity}

Room temperature electrical conductivity as well as the carrier concentration and mobility were characterized using van der pauw method.

Table III shows the sputtered thin film electrical properties from samples annealed at selected temperatures. The relative uncertainties of carrier concentration, Hall mobility,
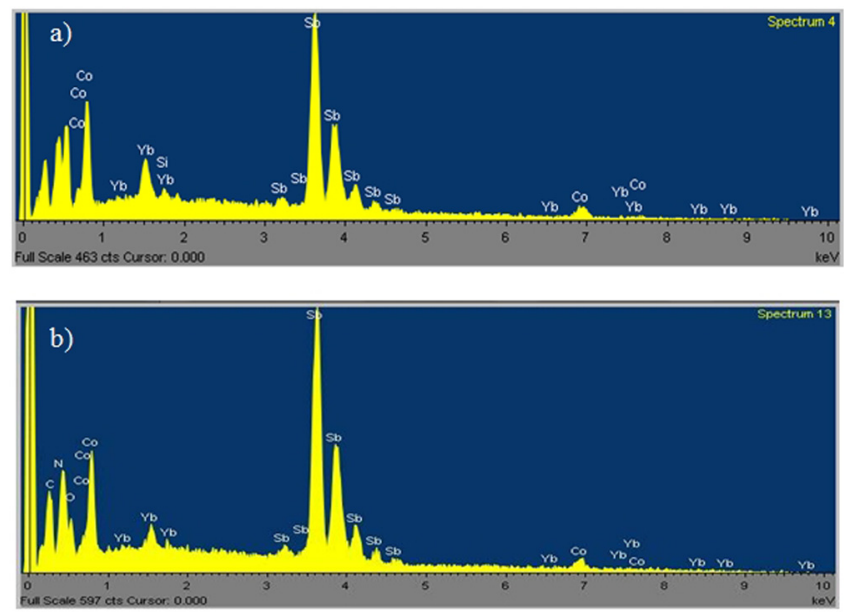

FIG. 2. EDS of as-sputtered filled skutterudite thin film on Si substrate sample (a) Y1 and (b) Y4. 
TABLE III. Room temperature Hall Effect for the $860 \mathrm{~nm}$ thin film at different heat treatment temperatures.

\begin{tabular}{lccc}
\hline \hline $\begin{array}{l}\text { Sample } \\
\text { No. }\end{array}$ & $\begin{array}{c}\text { Carrier } \\
\text { concentration } n\left(10^{18} / \mathrm{cm}^{3}\right)\end{array}$ & $\begin{array}{c}\text { Hall mobility } \\
\mu\left(\mathrm{cm}^{2} / \mathrm{V} \cdot \mathrm{s}\right)\end{array}$ & $\begin{array}{c}\text { Conductivity } \\
\sigma=n e \mu\left(10^{4} \mathrm{~S} / \mathrm{m}\right)\end{array}$ \\
\hline Y1 & 140000 & 0.07 & 16 \\
$\mathrm{Y} 2$ & 5000 & 1.5 & 12 \\
$\mathrm{Y} 3$ & 12 & 7.6 & 0.15 \\
$\mathrm{Y} 4$ & 8.3 & 89 & 1.2 \\
\hline \hline
\end{tabular}

and conductivity measurement are $10 \%, 2 \%$, and $8 \%$. After sputtering, the thin film samples show a very high electrical conductivity due to high carrier concentration. This is because the bonding between $\mathrm{Co}$ and $\mathrm{Sb}$ has not been fully formed which gives high carrier concentrations, while the mobility $0.07 \mathrm{~cm}^{2} / \mathrm{V}$ s is very low. After a $620 \mathrm{~K}$ heat treatment for $2 \mathrm{~h}$ (Y2), the $\mathrm{CoSb}_{3}$ phase starts to form as seen in Fig. 1 and mobility increases to $1.5 \mathrm{~cm}^{2} / \mathrm{V}$ s. Higher temperature heat treatments (Y3 and Y4) increase the mobility even more with some sacrifice of carrier concentration. Lower carrier concentration is beneficial to Seebeck coefficient as shown in Eq. (1). In fact, $1020 \mathrm{~K}$ heat treatment (Y4) fully crystallizes the sputtered thin film ${ }^{21}$ and more importantly has large carrier mobility. As a result, from now on an annealing temperature of $1020 \mathrm{~K}$ heat treatment will be used for our work.

Thin films of different thicknesses have been deposited using a DC magnetron sputtering machine with a constant sputtering rate. Hall Effect measurements reveal that carrier concentration and mobility both play important roles in electrical conductivity.
Electrical conductivity measurements for samples heat treated at $1020 \mathrm{~K}$ at temperatures of 300 to $770 \mathrm{~K}$ in vacuum are shown in Fig. 4. The measured temperature dependent electrical conductivities for sputtered thin film are shown in Fig. 4 with a relative uncertainty of $6 \%$. As can be seen, the variation in electrical conductivity is significant in samples of different thicknesses. The $130 \mathrm{~nm}$ thin film (Y6) has the smallest electrical conductivity due to its lower carrier concentration. The $300 \mathrm{~nm}$ and $860 \mathrm{~nm}$ thin films (Y5 and Y4) have higher conductivities with a higher carrier concentration level.

\section{Seebeck coefficient}

Fig. 5 shows the measured Seebeck coefficients for filled skutterudite thin films with various film thicknesses. Seebeck coefficients were measured using a differential method under vacuum by an MMR Seebeck S100 controller with a K20 temperature controller. Different from measurement of bulk material Seebeck coefficient, thin film material with $\mathrm{SiO}_{2}$ insulation layer and $\mathrm{Si}$ substrate has been cut into a long bar shape $(1 \times 1 \times 4 \mathrm{~mm})$. The reference material used for measurement is constantan wire provided by the same company. By putting reference material and thin film material under same temperature difference, the generated voltages are recorded automatically by the machine. The electrical contact between the measurement stage and thin film sample is only at thermoelectric material layer in order to eliminate any Seebeck coefficient that may generate from Si substrate. Seebeck coefficient is an intrinsic property that associated with the material and can be calculated as $\Delta \mathrm{V} / \Delta \mathrm{T}$. The reference material constantan has a known Seebeck coefficient, and the Seebeck of thin film material could be inferred from
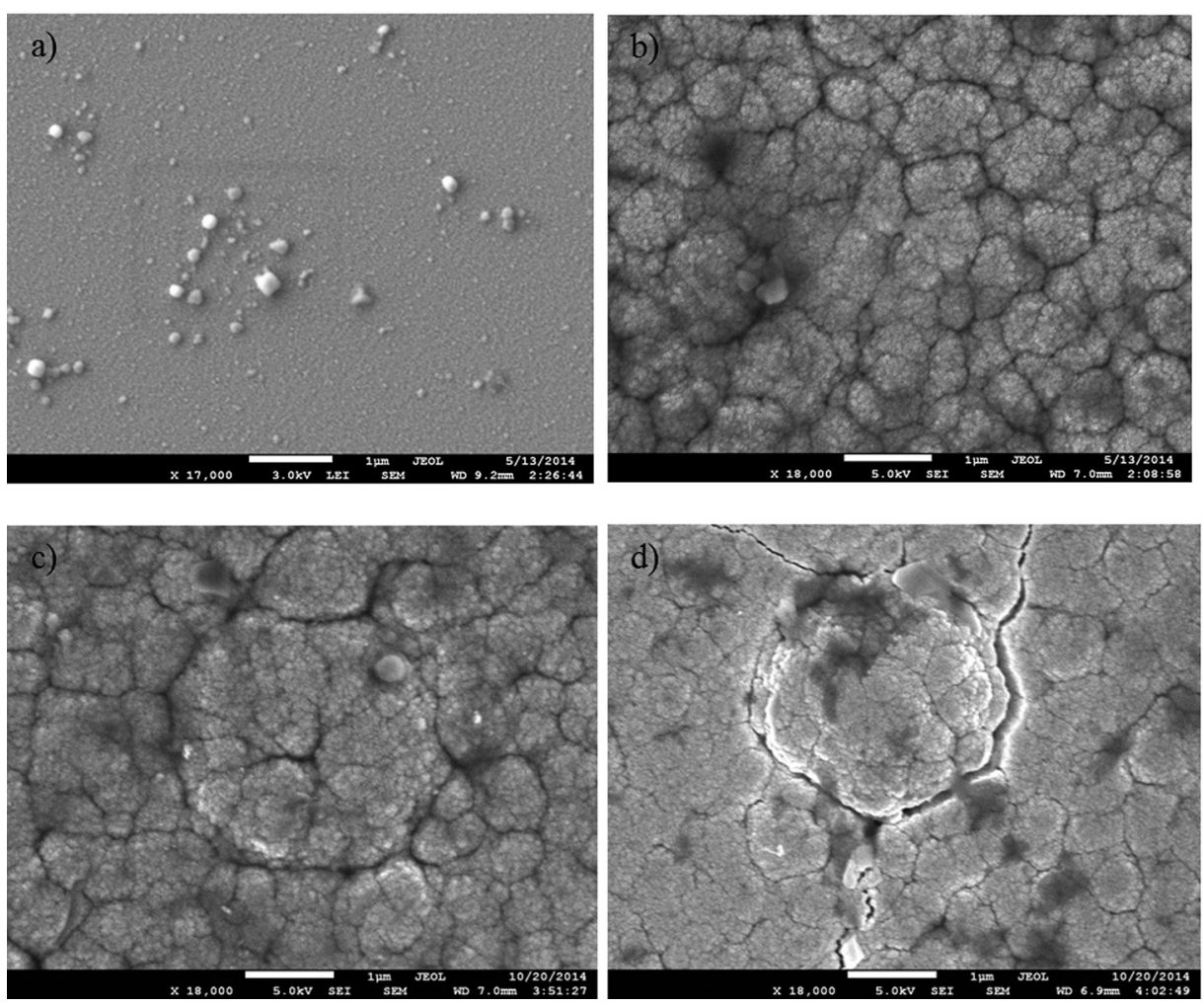

FIG. 3. SEM surface image of filled skutterudite thin film (a) as deposited Y1 sample, (b) $620 \mathrm{~K}$ heat treated Y2 sample, (c) $870 \mathrm{~K}$ HT Y3 sample, and (d) $1020 \mathrm{~K}$ HT Y4 sample. 


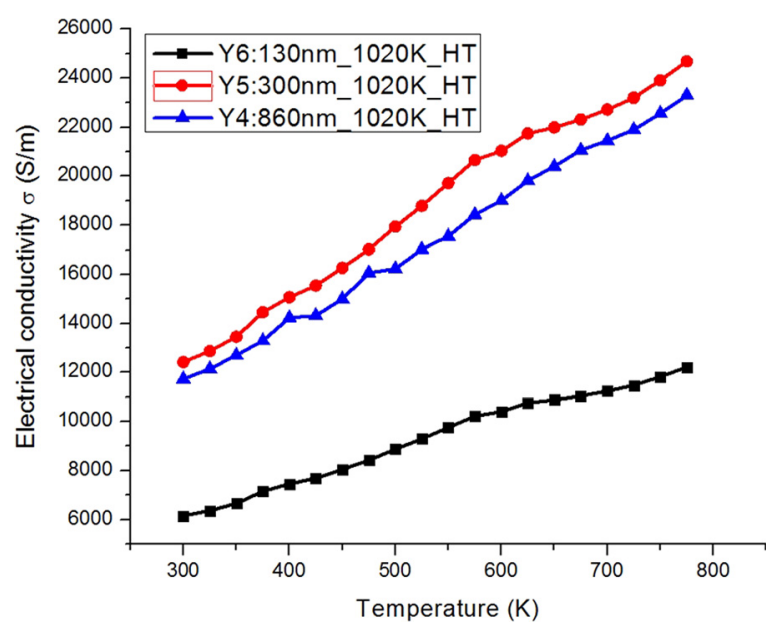

FIG. 4. Electrical conductivity of $1020 \mathrm{~K}$ heat treatment $\mathrm{Yb}$ doped $\mathrm{CoSb}_{3}$ thin films with different thicknesses.

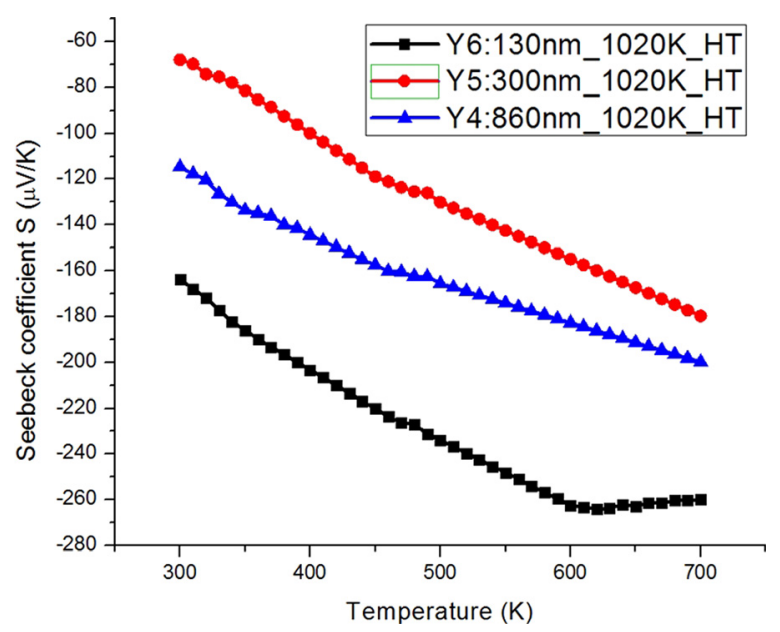

FIG. 5. Seebeck of $1020 \mathrm{~K}$ heat treated $\mathrm{Yb}$ doped $\mathrm{CoSb}_{3}$ thin films with different thicknesses.

ratio of voltage drop between reference material and sample under same temperature difference. The relative uncertainty of measured Seebeck coefficient is $9 \%$. The Seebeck coefficient is very sensitive to carrier concentration and is inversely proportional to electrical conductivity due to the opposite dependence of carrier concentration, i.e., $S \sim \ln (n)$ in Eq. (1). ${ }^{30}$ Fig. 5 shows that the $130 \mathrm{~nm}$ thick thin film (Y6) has a higher Seebeck coefficient because of its lower carrier concentration as shown in Table IV. As carrier concentration increases in Y4 and Y5 sample, the Seebeck

TABLE IV. Room temperature Hall Effect for $1020 \mathrm{~K}$ heat treated thin films at different thicknesses.

\begin{tabular}{lcccc}
\hline \hline Sample & $\begin{array}{c}\text { Thickness } \\
(\mathrm{nm})\end{array}$ & $\begin{array}{c}\text { Carrier } \\
\text { concentration } \\
n\left(10^{18} / \mathrm{cm}^{3}\right)\end{array}$ & $\begin{array}{c}\text { Hall mobility } \\
\mu\left(\mathrm{cm}^{2} / \mathrm{V} \mathrm{s}\right)\end{array}$ & $\begin{array}{c}\text { Conductivity } \\
\sigma=\text { ne } \mu\left(10^{4} \mathrm{~S} / \mathrm{m}\right)\end{array}$ \\
\hline Y4 & 860 & 8.3 & 89 & 1.2 \\
Y5 & 300 & 17 & 46 & 1.3 \\
Y6 & 130 & 4.8 & 80 & 0.61 \\
Anno $^{21}$ & 71 & 0.4 & 264 & 0.62 \\
\hline \hline
\end{tabular}

coefficient starts to decrease. Generally as temperature increases, the Seebeck coefficient will increase in magnitude because the ratio of effective density of state to carrier concentration $N_{c} / n$ in Eq. (1) is proportional to $\exp (\mathrm{kT})$. However, for $130 \mathrm{~nm}$ thin film (Y6) sample, the Seebeck coefficient starts to decrease around $600 \mathrm{~K}$ which may due to the carrier energy filtering effect that has been caused by the potential barriers at grain boundaries. ${ }^{21}$ The fact that the Seebeck coefficient is negative in Fig. 5 indicates that $\mathrm{Yb}$ doped $\mathrm{CoSb}_{3}$ thin film is an $n$-type semiconductor, which also agrees with the Hall Effect measurement.

\section{Thermal conductivity}

One popular technique in the thermal conductivity measurement is a $3 \omega$-method, in which a thin metal strip evaporated on the sample acts as a heat source and a thermometer. Thermal conductivity was measured though the $3 \omega$ method (invented by Cahill et $a l^{32,33}$ ) from room temperature up to $700 \mathrm{~K}$ by depositing Au strip on top of sample. The heater is driven with $\mathrm{AC}$ current at frequency $\omega$, which causes the electrical resistance of heat source to oscillate at frequency $2 \omega$. By monitoring the AC voltage as a function of the frequency of the applied AC current, thermal conductivity can be determined. Prior to the Au strip deposition, a thin of silicon oxide layer is grown by PECVD for electrical insulation.

The calculations of the thermal conductivity $k_{f i l m}$ of the thin films are carried out from the experimental data plotted in Fig. 6(b) in accordance with the method used by Lee and Cahil $^{34}$ using a simplified one-dimensional model

$$
k_{f i l m}=\frac{P_{l} \cdot t}{w \cdot \Delta T_{f i l m}},
$$

where $P_{l}$ is the supplied power per unit length of the narrow metal heater thermometer of the width $w, t$ is the thickness of the tested thin film, $\Delta T_{\text {film }}$ is the increase of the temperature oscillations due to the thin film with respect to the reference sample. Fig. 6 illustrates typical frequency dependent temperature oscillations in the sample containing the $\mathrm{Yb}$ filled $\mathrm{CoSb}_{3}$ thin film and reference samples. The calculation of the thermal conductivity using Eq. (2) gives a value of about $2-3 \mathrm{~W} / \mathrm{mK}$ at room temperature, which is 2 to 3 times lower than for the bulk material.

Temperature dependent thermal conductivity is measured similarly using $3 \omega$ method with the example shown in Fig. 6. The relative uncertainty of measured temperature dependent thermal conductivity is $15 \%$. At each temperature point, a frequency sweep is needed for the $\Delta T_{\text {film }}$ calculation for both thin film sample and reference sample. The TCR of e-beam evaporated gold is very stable up to $700 \mathrm{~K}$ and the resistivity can be calculated based on the measured TCR and room temperature resistivity. By applying Eq. (2), thermal conductivity was calculated and plotted in Fig. 7 for the thin films with different thicknesses. As temperature increases, all three films tend to decrease in thermal conductivity because phonon scattering is enhanced at higher temperatures. The thinner samples of $130 \mathrm{~nm}$ and $300 \mathrm{~nm}$ (Y6 and Y5) have a slightly lower thermal conductivity than the $860 \mathrm{~nm}$ thin film 

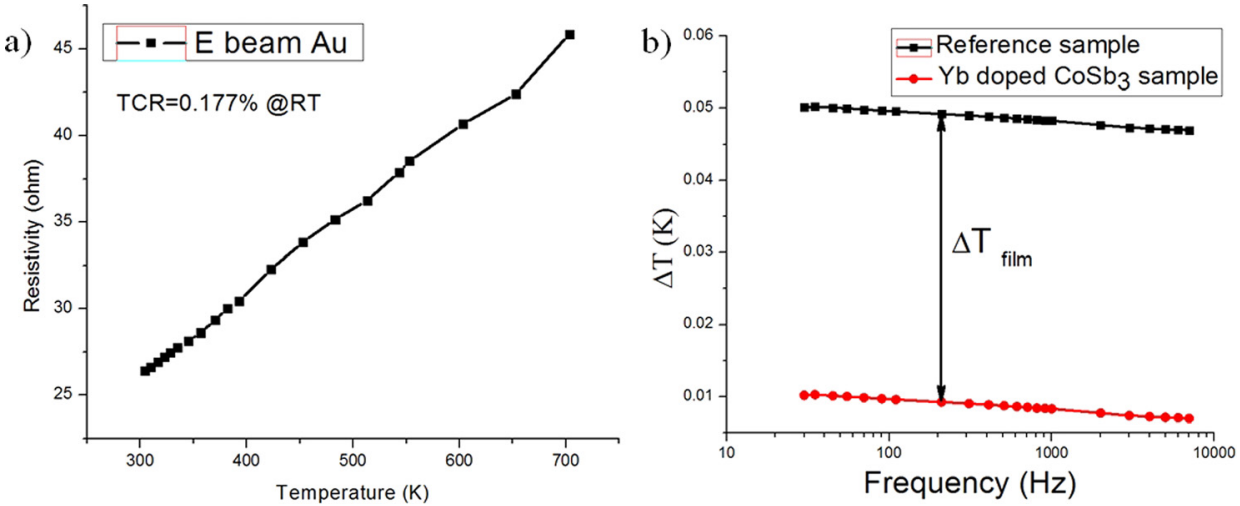

FIG. 6. Thermal conductivity measurement including (a)TCR of Au and (b) example of temperature oscillations, $\Delta \mathrm{T}$, measured in the $3 \omega$ method at $300 \mathrm{~K}$ on the $\mathrm{Yb}$ doped $\mathrm{CoSb}_{3}$ film and the reference sample.

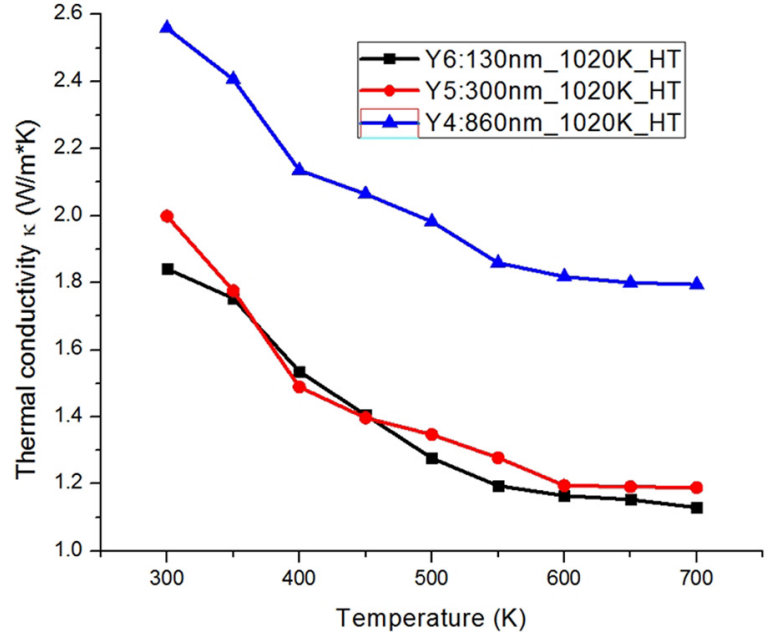

FIG. 7. Thermal conductivity of $\mathrm{Yb}$ doped $\mathrm{CoSb}_{3}$ film at different thicknesses with temperature dependent.

(Y4) which may be due to the enhanced phonon scattering by grain interface and boundary in thin films.

\section{Figure of merit, ZT}

The figure of merit $Z T$ value of the sample can be estimated by combining the electrical properties, Seebeck coefficient, and thermal conductivity. Fig. 8 shows the $Z T$ for $\mathrm{Yb}$

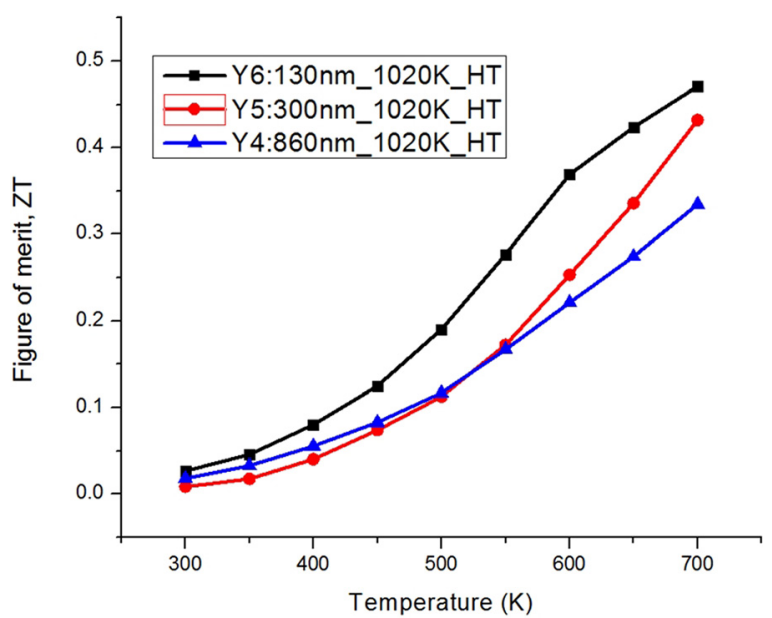

FIG. 8. Figure of Merit, $Z T$ of $\mathrm{Yb}$ filled $\mathrm{CoSb}_{3}$ films with different thicknesses. filled $\mathrm{CoSb}_{3}$ thin film with different thicknesses. The highest $Z T$ is achieved with the $130 \mathrm{~nm}$ thick sample (Y6) because it has much higher Seebeck coefficient even if its electrical conductivity is slightly lower than the other samples. Also, the thermal conductivity of the Y6 sample is lower than that of the Y4 sample. These results show the figure of merit does not significantly depend on the thin film thickness at lower temperatures. However, at each deposition and heat treatment, the carrier concentration and mobility do change a lot. Generally, with the same carrier concentration and mobility, thinner films may have higher figures of merit due to lower thermal conductivity caused by enhanced phonon scattering by boundaries.

Figures of merit of sputtered $\mathrm{Yb}$ doped $\mathrm{CoSb}_{3}$ in this work are found to be between half to one third of the bulk material reported by Nolas et al..$^{15}$ The lower $Z T$ in thin film mainly comes from the lower mobility. In bulk material, the mobility can be several thousand $\mathrm{cm}^{2} / \mathrm{V}$ s which is two orders of magnitude higher than thin film in this work. Higher temperature with longer heat treatment time may help increase the mobility but could also induce crack in the thin film if heating/cooling process is not well controlled.

\section{CONCLUSIONS}

Filled skutterudite is a state of the art high temperature thermoelectric material and in this work for the first time $\mathrm{Yb}$ filled $\mathrm{CoSb}_{3}$ skutterudite thin films have been reported using DC magnetron sputtering method. DC magnetron sputtering is adopted due to its high deposition rate and the maintenance of target material composition. More importantly, magnetron sputtering provides denser microstructure which surpasses most of the other thin film deposition techniques. The parameters of DC magnetron and post heat treatment on sputtered samples have been optimized experimentally and $\mathrm{Yb}$ filled $\mathrm{CoSb}_{3}$ thermoelectric properties have been characterized.

Electrical conductivity, Seebeck coefficient, thermal conductivity, and figure of merit are characterized from $300 \mathrm{~K}$ to $700 \mathrm{~K}$. XRD, SEM, and EDS of the different thicknesses samples at three kinds of heat treatment conditions are obtained to assess the phase composition and crystallinity of thin film samples under different heat treatment temperatures. In this work, $1020 \mathrm{~K}$ heat treatment was adopted for thin film post process due to the high degree of crystallinity as well as avoiding reverse heating effect. Carrier concentrations and 
Hall mobility are obtained from Hall Effect measurements, which provide further insight into the electrical conductivity and Seebeck coefficient mechanisms. The "as-sputtered" sample has a huge carrier concentration which gives very high electrical conductivity. However, low Hall mobility in the "as sputtered" sample makes it unsuitable for thermoelectric application. Among the three types of heat treatment, $1020 \mathrm{~K}$ gives the best Hall mobility. The Seebeck coefficient is inversely dependent on carrier concentration, so the low carrier concentration in the $130 \mathrm{~nm}$ sample increases the Seebeck coefficient significantly and overall gives the best figure of merit. Since the carrier concentration plays a tradeoff role in thermoelectric material, it should be optimized to give better ZT. It seems in this work that a low carrier concentration is more favorable due to its large increase in Seebeck coefficient when considering the square of Seebeck coefficient in the $Z T$ equation. Thermal conductivity of thin film filled skutterudite is found to be much less compared with bulk $\mathrm{Yb}$ filled $\mathrm{CoSb}_{3}$ skutterudite which may be due to the enhanced phonon scattering by grain interface and boundary. The $860 \mathrm{~nm}$ sample is much thicker than the rest two samples and has a higher thermal conductivity. Even though the fabrication setup for Y4, Y5, and Y6 are similar, the vacuum level and the Ar pressure during the sputtering condition is not exact same at each sputtering process which makes the electrical or thermal properties vary due to the change of carrier concentration, mobility, and defects shown in microstructure. It makes this work very hard to conclude a film thickness dependence of those measured electrical or thermal conductivity because of microstructure also change during heat treatment process. The electrical conductivity changes are explained by the measured carrier concentration and mobility for Y4, Y5, and Y6. Different thicknesses thin film samples are prepared with same sputtering deposition rate and maximum $Z T$ of 0.48 is achieved at $700 \mathrm{~K}$ for the $130 \mathrm{~nm}$ sample. This value is between half and one third of bulk figure of merit which is due to the lower Hall mobility in thin film samples.

\section{ACKNOWLEDGMENTS}

The authors gratefully acknowledge financial support from the NSF/DOE Thermoelectrics Partnership program under grant NSF CBET \#1048744. Research carried out in part at the Center for Functional Nanomaterials, Brookhaven National Laboratory, which is supported by the U.S. Department of Energy, Office of Basic Energy Sciences, under Contract No. DE-AC02-98CH10886. The authors wish to thank Dr. Xiaoya Shi and Dr. Fernando Camino of Brookhaven National Laboratory and Mr. Shuyu Wang and Mr. Shifeng Yu of Stony Brook University for help in fabrication and characterization of the samples. Special thanks go to Dr. Richard Gambino for the insightful discussions and James Kierstead for the manuscript improvements.

${ }^{1}$ C. Wood, Rep. Prog. Phys. 51(4), 459 (1988).

${ }^{2}$ G. J. Snyder and E. S. Toberer, Nature Mater. 7(2), 105 (2008).

${ }^{3}$ L. E. Bell, Science 321(5895), 1457 (2008).

${ }^{4}$ J. A. Paradiso and T. Starner, IEEE Pervas Comput. 4(1), 18 (2005).

${ }^{5}$ S. K. Lim, M. Y. Kim, and T. S. Oh, Thin Solid Films 517(14), 4199 (2009).

${ }^{6}$ R. J. McGlen, R. Jachuck, and S. Lin, Appl. Therm. Eng. 24(8-9), 1143 (2004).

${ }^{7}$ S. Lineykin and S. Ben-Yaakov, IEEE Trans. Ind. Appl. 43(2), 505 (2007).

${ }^{8}$ W. M. Yim and F. D. Rosi, Solid-State Electron. 15(10), 1121 (1972).

${ }^{9}$ I. Chowdhury, R. Prasher, K. Lofgreen, G. Chrysler, S. Narasimhan, R. Mahajan, D. Koester, R. Alley, and R. Venkatasubramanian, Nat. Nanotechnol. 4(4), 235 (2009).

${ }^{10} \mathrm{M}$. Stordeur and I. Stark, in Proceedings ICT'97-XVI International Conference on Thermoelectrics (1997), p. 575.

${ }^{11}$ M. S. Dresselhaus, G. Chen, M. Y. Tang, R. G. Yang, H. Lee, D. Z. Wang, Z. F. Ren, J. P. Fleurial, and P. Gogna, Adv. Mater. 19(8), 1043 (2007).

${ }^{12}$ D. Miorandi, S. Sicari, F. De Pellegrini, and I. Chlamtac, Ad Hoc. Networks 10(7), 1497 (2012).

${ }^{13}$ R. Venkatasubramanian, E. Siivola, T. Colpitts, and B. O'Quinn, Nature 413(6856), 597 (2001).

${ }^{14}$ G. S. Nolas, D. T. Morelli, and T. M. Tritt, Annu. Rev. Mater. Sci. 29, 89 (1999).

${ }^{15}$ G. S. Nolas, M. Kaeser, R. T. Littleton, and T. M. Tritt, Appl. Phys. Lett. 77(12), 1855 (2000)

${ }^{16}$ X. Shi, J. Yang, J. R. Salvador, M. F. Chi, J. Y. Cho, H. Wang, S. Q. Bai, J. H. Yang, W. Q. Zhang, and L. D. Chen, J. Am. Chem. Soc. 134(5), 2842 (2012).

${ }^{17}$ R. Venkatasubramanian, B. O'Quinn, E. Siivola, K. Coonley, P. Addepally, M. Napier, and T. Colpitts, Mater. Res. Soc. Symp. Proc. 793, 51 (2004).

${ }^{18}$ L. Xu, Y. C. Liu, B. B. Chen, C. Zhao, and K. Lu, Polym. Compos. 34(10), 1728 (2013).

${ }^{19}$ M. Y. Kim and T. S. Oh, J. Electron. Mater. 42(9), 2752 (2013).

${ }^{20}$ H. Anno, K. Matsubara, Y. Notohara, T. Sakakibara, K. Kishimoto, and T. Koyanagi, presented at the Fifteenth International Conference on Thermoelectrics, 1996.

${ }^{21}$ H. Anno, T. Sakakibara, Y. Notohara, H. Tashiro, T. Koyanagi, H. Kaneko, and K. Matsubara, presented at the Proceedings ICT'97. XVI International Conference on Thermoelectrics, 1997.

${ }^{22}$ V. Savchuk, A. Boulouz, S. Chakraborty, J. Schumann, and H. Vinzelberg, J. Appl. Phys. 92(9), 5319 (2002).

${ }^{23}$ J. C. Caylor, A. M. Stacy, R. Gronsky, and T. Sands, J. Appl. Phys. 89, $3508(2001)$

${ }^{24}$ R. Zeipl, J. Navratil, L. Benes, T. Kocourek, M. Jelinek, J. Lorinik, J. Vanis, J. Zelinka, and J. Walachova, in Proceedings of ICT'06: XXV International Conference on Thermoelectrics (2006), p. 451.

${ }^{25}$ R. Zeipl, J. Walachova, J. Lorincik, S. Leshkov, M. Josiekova, M. Jelinek, T. Kocourek, K. Jurek, J. Navratil, L. Benes, and T. Plechacek, J. Vac. Sci. Technol. A 28(4), 523 (2010).

${ }^{26}$ S. R. S. Kumar, A. Alyamani, J. W. Graff, T. M. Tritt, and H. N. Alshareef, J. Mater. Res. 26(15), 1836 (2011).

${ }^{27}$ B. G. Lewis and D. C. Paine, MRS Bull. 25(8), 22 (2000)

${ }^{28}$ P. J. Kelly and R. D. Arnell, Vacuum 56(3), 159 (2000).

${ }^{29}$ G. Min, D. M. Rowe, and K. Kontostavlakis, J. Phys. D: Appl. Phys. 37(8), 1301 (2004).

${ }^{30}$ G. Busch and U. Winkler, Helv. Phys. Acta 26(3-4), 395 (1953).

${ }^{31}$ C. Jacoboni and E. W. Prohofsky, Phys. Rev. B 1(2), 697 (1970).

${ }^{32}$ D. G. Cahill, Rev. Sci. Instrum. 61(2), 802 (1990).

${ }^{33}$ D. G. Cahill, Rev. Sci. Instrum. 73(10), 3701 (2002).

${ }^{34}$ S. M. Lee, D. G. Cahill, and T. H. Allen, Phys. Rev. B 52(1), 253 (1995). 